\title{
On Inequalities of Friedrichs and Babuška-Aziz in Dimension Three
}

\author{
W. Velte
}

\begin{abstract}
In 1983, Horgan and Payne have published a paper entitled "On inequalities of Korn, Friedrichs and Babuška-Aziz". In that paper the two authors have proven a simple relation between the optimal constants occuring in the three inequalities under consideration. The analysis there is restricted to inequalities for functions of two variables only. In the present paper we will show that the results of Horgan and Payne concerning two of the inequalities have a counterpart in dimension three where, however, the situation is different in some aspects.
\end{abstract}

Keywords: Functional inequalities, optimal constants, eigenvalues, linear elasticity, Stokes equations

AMS subject classiflcation: 35 P 99, 35 Q 30, 35 Q 72

\section{Introduction}

In dimension two, Friedrichs' inequality reads as follows. Let $\Omega$ denote a bounded and simply connected open domain with boundary in the class $C^{1}$. Let $u, v$ denote pairs of square integrable conjugate harmonic functions defined on $\Omega$. Then (see [4]) there is a constant $\Gamma$ depending only on the shape of the region $\Omega$ such that

$$
\int_{\Omega} u^{2} d A \leq \Gamma \int_{\Omega} v^{2} d A, \quad \text { provided that } \quad \int_{\Omega} u d A=0,
$$

and

$$
\int_{\Omega} v^{2} d A \leq \Gamma \int_{\Omega} u^{2} d A, \quad \text { provided that } \quad \int_{\Omega} v d A=0 .
$$

To formulate the inequality of Babuška-Aziz, we have to introduce the real function spaces $L^{2}(\Omega)$ and $H_{0}^{1}(\Omega)$. Here, $L^{2}(\Omega)$ denotes the linear space of all square integrable real functions defined on $\Omega$, which is a Hilbert space with respect to the scalar product $(u, v)_{0}=\int_{\Omega} u v d A$ and the associated norm. $H_{0}^{1}(\Omega)$ denotes the Sobolev space consisting of all real functions $u$ which belong to $L^{2}(\Omega)$ together with their first (generalized) partial derivatives $u, k(k=1,2)$, and which vanish at the boundary.

Then, given any function $p \in L^{2}(\Omega)$ satisfying $\int_{\Omega} p d A=0$, there is a vector function $w=\left(w_{1}, w_{2}\right)$ with components $w_{1}, w_{2} \in H_{0}^{1}(\Omega)$ and a constant $C$ depending only on

W. Velte: University of Würzburg, Inst. Appl. Math. and Stat., Am Hubland, D - 97074 Würzburg 
the shape of $\Omega$ such that $\operatorname{div} w=p$ and

$$
D(w)=\sum_{j, k=1}^{2} \int_{\Omega} w_{j, k} w_{j, k} d A \leq C \int_{\Omega}(\operatorname{div} w)^{2} d A
$$

(See [1: pp. $172-174]$. A proof of the inequality in dimension three may be found in Ladyzhenskaya and Solonnikov [9: pp. 265 - 266].)

In [6], Horgan and Payne have shown in particular that the optimal constants in the above inequalities are related by

$$
\Gamma=C-1
$$

For further results obtained by Horgan and Payne we refer to [6] and to Horgan's review paper [7], and the references given there.

In the present paper we will deal with analogous inequalities for functions of three variables. $\Omega$ will now denote a three-dimensional bounded and simply-connected open domain. Unless otherwise specified, the boundary of $\Omega$ is assumed to be in the class $C^{2}$.

Pairs $u, v$ of conjugatc harmonic functions are now replaced by pairs of functions $p$ and vector-valued functions $q=\left(q_{1}, q_{2}, q_{3}\right)$ satisfying the equations

$$
\operatorname{rot} q=-\nabla p \quad \text { and } \quad \operatorname{div} q=0 \text {. }
$$

In quaternionic analysis the latter are known as equations of Moisil-Teodorescu. As we will see, there are now two constants $\Gamma$ and $\tilde{\Gamma}$ depending only on the shape of the domain $\Omega$ such that for all sqare integrable solutions of equations (1) the following inequalities hold:

$$
\int_{\Omega} p^{2} d V \leq \Gamma \int_{\Omega}|q|^{2} d V, \quad \text { provided that } \quad \int_{\Omega} p d V=0
$$

and

$$
\int_{\Omega}|q|^{2} d V \leq \tilde{\Gamma} \int_{\Omega} p^{2} d V, \quad \text { provided that } \quad q \cdot n=0 \text { on } \partial \Omega .
$$

In the constraint $q \cdot n=0, n$ denotes the outer unit normal to the boundary.

Also in the space $H_{0}^{1}(\Omega)$ there are now two inequalities, namely

$$
D(w) \leq C \int_{\Omega}(\operatorname{div} w)^{2} d V \quad \text { for } \quad w \in \operatorname{Ker} \operatorname{div}^{\perp}
$$

and

$$
D(w) \leq \tilde{C} \int_{\Omega}|\operatorname{rot} w|^{2} d V \cdot \text { for } w \in \operatorname{Kerrot}^{\perp} .
$$

Here, Ker div and Ker rot denote the subspaces of all functions satisfying $\operatorname{div} w=0$ and $\operatorname{rot} w=0$, respectively, whereas $\operatorname{Kerdiv}^{\perp}$ and $\operatorname{Ker} \operatorname{rot}^{\perp}$ are their orthogonal complements in $H_{0}^{1}(\Omega)^{3}$ with respect to the scalar product

$$
D(w, \tilde{w})=\sum_{j, k=1}^{3} \int_{\Omega} w_{j, k} \tilde{w}_{j, k} d V
$$


In Section 1 of this paper we will show that the optimal constants are related by

$$
\Gamma=C-1 \quad \text { and } \quad \tilde{\Gamma}=\tilde{C}-1
$$

Then, in Second 2, we will study the close relation between the optimal constants and the spectrum of two associated eigenvalue problems. One of them is an eigenvalue problem already studied by the Cosserats' [2] in the context of linear elasticity, whereas the other one is a counterpart to Friedrichs' eigenvalue problem treated in [4]. Among others we will show that

$$
\Gamma \geq 1 \quad \text { and } \quad \tilde{\Gamma} \geq 1
$$

for bounded and simply connected domains, and that $\Gamma=2$ and $\tilde{\Gamma}=1$ if $\Omega$ is a ball. In Section 3 we will exhibit a connection with the Stokes equations.

\section{Relations between the optimal constants}

In what follows, our analysis rests upon results which are partly valid for bounded domains with Lipschitz-continuous boundary. We will, however, also quote some results from Dautray and Lions [3] and from Mikhlin [10] which are established there only for domains with sufficiently smooth boundary. Details will be specified below.

Let us recall that the linear space $C_{0}^{\infty}(\Omega)$, often also denoted by $\mathcal{D}(\Omega)$, consists of all infinitely differentable functions with compact support in $\Omega$, whereas $H_{0}^{1}(\Omega)$ is by definition the closure of $C_{0}^{\infty}(\Omega)$ in the Sobolev space $H^{1}(\Omega)$. As is well known, we may introduce in $H_{0}^{1}(\Omega)$ also

$$
(u, v)_{1}=\sum_{k=1}^{3} \int_{\Omega} u, k v, k d V \quad \text { and } \quad|u|_{1}=(u, u)_{1}^{1 / 2}
$$

where the norm $|\cdot|_{1}$ is equivalent to the usual Sobolev norm $\|\cdot\|_{1}$. Analogously we introduce in $H_{0}^{1}(\Omega)^{3}$ the forms $D(\cdot, \cdot)$ and $D(\cdot)$ already defined above. Clearly, $H_{0}^{1}(\Omega)^{3}$ is a Hilbert space with respect to $D(\cdot, \cdot)$ and $D(\cdot)^{1 / 2}$.

In our analysis the following results will play a basic role, where we refer to Girault and Raviart [5] for the operator div and to Dautray and Lions [3] for the Operator rot.

Proposition 1 (see [5: p. 24]). Let $\Omega$ denote a bounded and connected domain with Lipschitz-continuous boundary. Then the operator div maps $H_{0}^{1}(\Omega)^{3}$ onto the the space $L_{0}^{2}(\Omega)$, where

$$
L_{0}^{2}(\Omega)=\left\{p \in L^{2}(\Omega) \mid \int_{\Omega} p d V=0\right\} .
$$

Let us now consider the image under the operator rot. If the connected domain $\Omega$ is not simply-connected, then the result depends on the number of handles. For the sake of simplicity, however, we will restrict our attention to simply-connected domains. 
Proposition 2 (see [3: Chapter IX, p. 226]). Let $\Omega$ denote a bounded and simplyconnected open domain with boundary in the class $C^{2}$. Then the operator rot maps $H_{0}^{1}(\Omega)$ onto the closed linear space $H_{0}(\operatorname{div} 0, \Omega)$, where

$$
H_{0}(\operatorname{div} 0, \Omega)=\left\{q \in L^{2}(\Omega)^{3} \mid \operatorname{div} q=0 \text { and } q \cdot n=0\right\} .
$$

Here $\operatorname{div} q=0$ together with $q \cdot n=0$ is satisfied in the weak sense

$$
\int_{\Omega} q \nabla \Phi d V=0 \quad \text { for all } \Phi \in C_{0}^{\infty}\left(\mathbb{R}^{3}\right) .
$$

We will make use of the fundamental decomposition of $L^{2}(\Omega)^{3}$, namely

$$
L^{2}(\Omega)^{3}=\operatorname{grad} H^{1}(\Omega) \oplus H_{0}(\operatorname{div} 0, \Omega)
$$

(See, for instance, [3: Chapter IX, p.216].)

We now turn to an orthogonal decomposition of the space $H_{0}^{1}(\Omega)^{3}$. It rests upon the identity

$$
D(w, \tilde{w})=d(w, \tilde{w})+r(w, \tilde{w}) \quad \text { for } \quad w, \tilde{w} \in H_{0}^{1}(\Omega)^{3},
$$

where, by definition,

$$
d(w, \tilde{w})=\int_{\Omega} \operatorname{div} w \operatorname{div} \tilde{w} d V \quad \text { and } \quad r(w, \tilde{w})=\int_{\Omega} \operatorname{rot} w \cdot \operatorname{rot} \tilde{w} d V .
$$

On partial integration, identity (4) is easily verified for functions $w, \tilde{w} \in C_{0}^{\infty}(\Omega)^{3}$. The latter space, however, is dense in $H_{0}^{1}(\Omega)^{3}$.

From the obvious inequalities $d(w) \leq D(w)$ and $r(w) \leq D(w)$ there follows that Ker div and Ker rot are closed linear subspaces of $H_{0}^{1}(\Omega)^{3}$. Hence we may decompose $H_{0}^{1}(\Omega)^{3}$ into three $D(\cdot, \cdot)$-orthogonal subspaces, namely

$$
H_{0}^{1}(\Omega)^{3}=\operatorname{Ker} \operatorname{div} \oplus \operatorname{Ker} \operatorname{rot} \oplus W
$$

Note that the three subspaces are also pairwise orthogonal with respect to the bilinear forms $d(\cdot, \cdot)$ and $r(\cdot, \cdot)$ introduced above.

From Propositions 1 and 2 it is clear that the operators div and rot are one-to-one as mappings of Ker div ${ }^{\perp}$ onto $L_{0}^{2}(\Omega)$ and of $\operatorname{Ker}_{\operatorname{rot}^{\perp}}^{\perp}$ onto $H_{0}(\operatorname{div} 0, \Omega)$, respectively. But, since all these spaces are Hilbert spaces as well, it follows from Banach's well known inverse operator theorem that the inverses of div and rot are bounded linear operators, too. Thus one obtains the following

Proposition 3. Let $\Omega$ denote a bounded and simply-connected open domain with boundary in the class $C^{2}$. Then there are constants $C$ and $\tilde{C}$ such that

$$
D(w) \leq C d(w) \quad \text { for all } \quad w \in W \oplus \text { Ker rot }
$$

and

$$
D(w) \leq \tilde{C} r(w) \quad \text { for all } \quad w \in W \oplus \operatorname{Ker} \operatorname{div}
$$


whence due to (4)

$$
r(w) \leq(C-1) d(w) \text { and } d(w) \leq(\tilde{C}-1) r(w) \quad \text { for all } w \in W .
$$

Thus $D(\cdot)^{1 / 2}, d(\cdot)^{1 / 2}$, and $r(\cdot)^{1 / 2}$ are equivalent norms in the subspace $W$, and the optimal constants in the inequalities (6) and (7) satisfy $C>1$ and $\tilde{C}>1$, respectively.

Let us now consider equations (1). They may be written in weak form as follows. Find functions $p \in L^{2}(\Omega)$ and $q \in L^{2}(\Omega)^{3}$ satisfying the variational equations

$$
\int_{\Omega} q \operatorname{rot} \phi d V=\int_{\Omega} p \operatorname{div} \phi d V \quad \text { for all } \phi \in C_{0}^{\infty}(\Omega)^{3}
$$

and

$$
\int_{\Omega} q \nabla \Phi d V=0 \quad \text { for all } \Phi \in C_{0}^{\infty}(\Omega)
$$

Inserting functions $\phi=\nabla \Phi, \Phi \in C_{0}^{\infty}(\Omega)$ into equation (8), one obtains

$$
\int_{\Omega} p \triangle \Phi d V=0 \quad \text { for all } \Phi \in C_{0}^{\infty}(\Omega) \text {. }
$$

Hence, by Weyl's Lemma, $p$ is equivalent to a harmonic function, that is $p \in C^{\infty}(\Omega)$, $\Delta p=0$ in $\Omega$. Inserting instead functions $\phi=\operatorname{rot} \psi, \Phi=\operatorname{div} \psi, \psi \in C_{0}^{\infty}(\Omega)^{3}$ into (8) and (9), one gets

$$
\int_{\Omega} q \operatorname{rot} \operatorname{rot} \psi d V=0 \quad \text { and } \quad \int_{\Omega} q \nabla \operatorname{div} \psi d V=0, \quad \text { whence } \int_{\Omega} q \Delta \psi d V=0
$$

for all $\psi \in C_{0}^{\infty}(\Omega)^{3}$. (Here we have used $\nabla \operatorname{div}=\Delta+$ rot rot.) Again by Weyl's Lemma; one obtains $q \in C^{\infty}(\Omega)^{3}, \Delta q=0$ in $\Omega$. Thus we see that any pair $(p, q) \epsilon$ $L^{2}(\Omega) \times L^{2}(\Omega)^{3}$ satisfying the variational equations is solution of equations (1) in the classical sense, too.

If the pair $p, q$ is a solution of (8), (9), then the same holds for the pair $p+c, q+\nabla h$ where $c$ denotes any real constant and $h$ any harmonic function belonging to $H^{1}(\Omega)$. There is a constant $c$ such that $p+c$ has mean value zero, and there is a uniquely determined $\nabla h$ such that $q+\nabla h \in H_{0}(\operatorname{div} 0, \Omega)$. Indeed, due to the orthogonal decomposition (3) we may write $q=-\nabla h+q_{0}$, where $h \in H^{1}(\Omega)$ and $q_{0} \in H_{0}(\operatorname{div} 0, \Omega)$. On insertin $g q$ into equation (9) we conclude by Weyl's lemma that $h$ is harmonic and $q+\nabla h=q_{0} \in H_{0}(\operatorname{div} 0, \Omega)$.

Thus we may restrict our attention to normalized solutions, that is we may look for solutions $p$ and $q$; where $p$ belongs to the linear space $L_{0}^{2}(\Omega)$, and $q$ to the linear space $H_{0}(\operatorname{div} 0, \Omega)$. These functions $p$ and $q$, however, satisfy due to the orthogonal decomposition (3) the inequalities

$$
\int_{\Omega} p^{2} d V \leq \int_{\Omega}(p+c)^{2} d V \quad \text { and } \quad \int_{\Omega}|q|^{2} d V \leq \int_{\Omega}|q+\nabla h|^{2} d V
$$

for any constant $c$ and any harmonic function $h \in H^{1}(\Omega)$.

So far we have seen that any solution $p, q$ of the equations (8), (9) is harmonic, and that it can be normalized imposing the constraints $\int_{\Omega} p d V=0$ and $q \cdot n=0$ on $\partial \Omega$. Let us now exhibit the relation between those solutions and the subspace $W$ introduced in the decomposition (5) of the space $H_{0}^{1}(\Omega)^{3}$. 


\section{Proposition 4.}

(a) Let $p, q$ be any solution of equations (8), (9). Then the following properties are equivalent:

(i) $p \in L_{0}^{2}(\Omega)$ and $q \in H_{0}(\operatorname{div} 0, \Omega)$.

(ii) $p \in \operatorname{div} W$ and $q \in \operatorname{rot} W$.

(b) To any given $p \in \operatorname{div} W$ there exists one and only one $q \in \operatorname{rot} W$, and to any given $q \in \operatorname{rot} W$ there exists one and only one $p \in \operatorname{div} W$ such that the pair $p, q$ is solution of $(8),(9)$.

Proof. (a) First let us recall that the three subspaces in the orthogonal decomposition (5) are also orthogonal with respect to the bilinear forms $d(\cdot, \cdot)$ and $r(\cdot, \cdot)$ so that

$$
L_{0}^{2}(\Omega)^{3}=\operatorname{div} H_{0}^{1}(\Omega)^{3}=\operatorname{div} W \oplus \operatorname{div} \operatorname{Ker} \operatorname{rot}
$$

and

$$
H_{0}(\operatorname{div} 0, \Omega)=\operatorname{rot} H_{0}^{1}(\Omega)^{3}=\operatorname{rot} W \oplus \operatorname{rot} \operatorname{Ker} \operatorname{div} .
$$

Let now $p, q$ denote any solution of (8), (9) satisfying conditions (i). Since $C_{0}^{\infty}(\Omega)$ is dense in $H_{0}^{l}(\Omega)$, equation (8) is satisfied in the form

$$
\int_{\Omega} q \operatorname{rot} \phi d V=\int_{\Omega} p \operatorname{div} \phi d V \quad \text { for all } \phi \in H_{0}^{1}(\Omega)^{3}
$$

as well. In particular there follows

$$
\int_{\Omega} p \operatorname{div} \phi d V=0 \quad \forall \phi \in \operatorname{Ker} \operatorname{rot} \quad \text { and } \quad \int_{\Omega} q \operatorname{rot} \phi d V=0 \quad \forall \phi \in \operatorname{Ker} \operatorname{div},
$$

whence $p \in \operatorname{div} W$ and $q \in \operatorname{rot} W$. The converse statement follows from Propositions 1 and 2.

(b) Let $p \in \operatorname{div} W$ be given and consider the variational problem to find $w \in W$ such that

$$
\int_{\Omega} \operatorname{rot} w \operatorname{rot} \phi d V=\int_{\Omega} p \operatorname{div} \phi d V \quad \text { for all } \phi \in W .
$$

Since, by Proposition 3 , the subspace $W$ is a Hilbert space also with respect to the scalar product $r(\cdot, \cdot)$ and the norm $r(\cdot)^{1 / 2}$, and since the linear functional on the right-hand side of (11) is bounded in the latter norm, it follows from Riesz's representation theorem that there exists a uniquely determined $w \in W$ satisfying equation (11). That equation, however, is then satisfied for all $\phi \in H_{0}^{1}(\Omega)^{3}$, too. On setting $q=\operatorname{rot} w$, the pair $p, q$ is a solution of (8), (9). For given $q \in \operatorname{rot} W$, consider now the variational problem to find $w \in W$ such that

$$
\int_{\Omega} \operatorname{div} w \operatorname{div} \phi d V=\int_{\Omega} q \operatorname{rot} \phi d V \quad \text { for all } \phi \in W .
$$

Then, again due to Proposition 3 and Riesz's representation theorem, there exists a uniquely determined function $w \in W$ satisfying (12) for all $\phi \in H_{0}^{1}(\Omega)^{3}$. On setting $p=\operatorname{div} w$, the pair $p, q$ is a solution of (8), (9) 
Theorem 1. Let $\Omega$ denote a three-dimensional bounded and simply-connected open domain with boundary in the class $C^{2}$. Then there are constants $\Gamma$ and $\tilde{\Gamma}$ depending only on the shape of $\Omega$ such that for any solution $p, q$ of the variational problem (8), (9) the following inequalities hold:

$$
\int_{\Omega} p^{2} d V \leq \Gamma \int_{\Omega}|q|^{2} d V \quad \text { provided that } \int_{\Omega} p d V=0
$$

and

$$
\int_{\Omega}|q|^{2} d V \leq \tilde{\Gamma} \int_{\Omega} p^{2} d V \quad \text { provided that } q \cdot n=0 \text { on } \partial \Omega .
$$

The optimal constants $\Gamma$ and $\tilde{\Gamma}$ in (13) and (14) are related to the optimal constants $C$ and $\tilde{C}$ in (6) and (7) by

$$
\Gamma=C-1 \quad \text { and } \quad \tilde{\Gamma}=\tilde{C}-1 .
$$

Proof. In view of (10) it is sufficient to consider solutions $p$ and $q$ normalized by the conditions $p \in L_{0}^{2}(\Omega)$ and $q \in H_{0}(\operatorname{div} 0, \Omega)$, so that $p \in \operatorname{div} W$ and $q \in \operatorname{rot} W$. Then, due to Proposition 3, one obtains

$$
\begin{aligned}
\left(\int_{\Omega} p^{2} d V\right)^{2} & =\left(\int_{\Omega} p \operatorname{div} w d V\right)^{2} \\
& =\left(\int_{\Omega} q \cdot \operatorname{rot} w d V\right)^{2} \\
& \leq(C-1) \int_{\Omega}|q|^{2} d V \int_{\Omega}|\operatorname{div} w|^{2} d V
\end{aligned}
$$

or

$$
\int_{\Omega} p^{2} d V \leq(C-1) \int_{\Omega}|q|^{2} d V \quad \text { and } \quad \Gamma \leq C-1
$$

Now, choose any $w \in W$ and set $q=\operatorname{rot} w$. By Proposition 4 there exists a conjugate function $p \in \operatorname{div} W$ satisfying (8), (9), and one finds

$$
\begin{aligned}
\left(\int_{\Omega}|q|^{2} d V\right)^{2} & =\left(\int_{\Omega} q \cdot \operatorname{rot} w d V\right)^{2} \\
& =\left(\int_{\Omega} p \operatorname{div} w d V\right)^{2} \\
& \leq \Gamma \int_{\Omega}|q|^{2} d V \int_{\Omega}(\operatorname{div} w)^{2} x d A
\end{aligned}
$$

or

$$
\int_{\Omega}|\operatorname{rot} w|^{2} d V \leq \Gamma \int_{\Omega}(\operatorname{div} w)^{2} d V \quad \text { and } \quad C-1 \leq \Gamma .
$$

On combining both results one concludes that $\Gamma=C-1$.

The proof of the relation $\tilde{\Gamma}=\tilde{C}-1$ is very similar. Given any solution $p$ and $q$, introduce $w \in W$ such that $q=\operatorname{rot} w$ to establish $\tilde{\Gamma} \leq \tilde{C}-1$. Given any $w \in W$, define $p=\operatorname{div} w$ and introduce the conjugate function $q \in \operatorname{rot} w$ to obtain $\tilde{C}-1 \leq \tilde{\Gamma}$ 


\section{Associated eigenvalue problems}

In dimension two, associated eigenvalue problems were already studied by Horgan (see [8]). Later on another approach has been used by the present author (see [11]). To treat now the three-dimensional problem, we will proceed similar as in [11]. To begin with, let us consider the following eigenvalue problem in variational form.

Problem 1. Find functions $w \in H_{0}^{1}(\Omega), w \neq 0$ and real numbers $\lambda$ such that

$$
D(w, \phi)=\lambda d(w, \phi) \quad \text { for all } \phi \in H_{0}^{1}(\Omega) .
$$

This is a weak form of the classical eigenvalue problem

$$
\left.\begin{array}{rl}
\triangle w-\lambda \nabla \operatorname{div} w=0 & \text { in } \Omega \\
w=0 & \text { on } \partial \Omega
\end{array}\right\} .
$$

Problem (17) plays a well known role in linear elasticity and is one of the problems already studied by the Cosserats' [2] one. hundred years ago. (For even earlier work see the references given there.) A modern and more complete treatment of problem (17) can be found in Mikhlin's work, where we refer to his survey paper [10] and the references quoted there. In Mikhlin's approach problem (17) is treated in operator form $A w=\lambda B w$ which, however, can be seen to be equivalent with the variational eigenvalue problem (16).

In view of (4), the eigenvalue problem (16) may be written in the form

$$
\int_{\Omega} \operatorname{rot} w \operatorname{rot} \phi d V=(\lambda-1) \int_{\Omega} \operatorname{div} w \operatorname{div} \phi d V \quad \text { for all } \phi \in H_{0}^{1}(\Omega)
$$

or, if $\lambda \neq 1$,

$$
\int_{\Omega} \operatorname{div} w \operatorname{div} \phi d V=(\lambda-1)^{-1} \int_{\Omega} \operatorname{rot} w \operatorname{rot} \phi d V \quad \text { for all } \phi \in H_{0}^{1}(\Omega) .
$$

From the orthogonal decomposition (5) it becomes clear that the eigenvalue problem splits into three independent problems in the pairwise orthogonal subspaces Ker rot, Ker div and $W$. Obviously, $\lambda=1$ is eigenvalue to the eigenspace Ker rot, whereas Ker div may be interpreted as eigenspace to the eigenvalue $\lambda=\infty$. In view of Proposition 3 we thus arrive to the following

\section{Proposition 5.}

(i) $\lambda=1$ and $\lambda=\infty$ are eigenvalues of infinite multiplicity to the eigenspaces Ker rot and Ker div, respectively.

(ii) For any other eigensolution $w, \lambda$ there holds $w \in W$ and

$$
(\tilde{C}-1)^{-1} \leq \lambda-1 \leq C-1,
$$

where $\tilde{C}>1$ and $C>1$ denote the optimal constants introduced in Proposition 9.

For bounded spherical and ellipsoidal domains $\Omega$ the structure of the eigenvalue spectrum was investigated by the Cosserats' [2]. As to the general case we quote some of Mikhlin's results from [10] as follows. 
Theorem 2 (Mikhlin). Let $\Omega$ be a bounded open domain with smooth boundary. Then there is a countable system $\left\{w_{k}\right\}$ of eigenfunctions $w_{k} \in H_{0}^{1}(\Omega)$ which are orthogonal with respect to $D(\cdot, \cdot)$ and complete in $H_{0}^{1}(\Omega)$. All eigenvalues $\lambda$ other than 1,2 and $\infty$ have finite multiplicity. They can accumulate only at $\lambda^{*}=2$.

Remark. In Mikhlin's paper, the boundary is supposed to be "sufficiently smooth". The assumption $C^{2}$ should be sufficient. But for the sake of easy reference we will adopt the more restrictive assumption $C^{\infty}$ throughout this section.

To illustrate the theorem we present one of the results of the Cosserats'. If $\Omega$ is a ball, then the eigenvalues $\lambda$ other than 1 and $\infty$ are given by

$$
\lambda_{n}=2+\frac{1}{n} \quad(n \in \mathbb{N})
$$

Here, $\lambda_{n}$ is eigenvalue of multiplicity $2 n+1$, and the eigenvalues accumulate at $\lambda^{*}=2$. In the case of spherical shells and ellipsoidal domains, the eigenvalue spectrum exhibits the same structure. But it seems to be an open question wether for other bounded domains $\lambda^{*}=2$ could be eigenvalue of infinite multiplicity, too.

On combining Proposition 5 and Theorem 2 we easily obtain the following

Theorem 3. Let $\Omega$ be a bounded and simply-connected domain with boundary in the class $C^{\infty}$. Then the optimal constants introduced in (6), (7) and (13),(14) satisfy

$$
\Gamma=C-1 \geq 1 \quad \text { and } \quad \tilde{\Gamma}=\tilde{C}-1 \geq 1 \text {. }
$$

The smallest intervall $[a, b]$ containing all eigenvalues $\lambda$ other than 1 and $\infty$ is related to the optimal constants by

$$
a=1+(\tilde{C}-1)^{-1} \quad \text { and } \quad b=C .
$$

If $\Omega$ is a ball, then

$$
\Gamma=2 \quad \text { and } \quad \tilde{\Gamma}=1 \text {. }
$$

Proof. Due to Theorem 2, there exists in $W$ a complete $D$-orthogonal system $\left\{w_{n}\right\}$ of eigenfunctions. They are orthogonal with respect to $d(\cdot, \cdot)$ and $r(\cdot, \cdot)$, too. In addition, $D(\cdot)^{1 / 2}$ and $d(\cdot)^{1 / 2}$ are equivalent norms in $W$. Thus we may assume that the eigenfunctions $w_{n}$ are normalized by $d\left(w_{n}\right)=1$. Then any function $w \in W, w \neq 0$ has a representation

$$
w=\sum_{n} c_{n} w_{n} \quad \text { with } \quad c_{n}=d\left(w, w_{n}\right)
$$

(summation from 1 to $\infty$ ) and one obtains

$$
\frac{r(w)}{d(w)}=\frac{\sum_{n}\left(\lambda_{k}-1\right) c_{n}^{2}}{\sum_{n} c_{n}^{2}}
$$

which yields (21). Since the point of accumulation $\lambda^{*}=2$ is contained in the interval $[a, b]$, we infer in particular (20). If the domain $\Omega$ is a ball, then $a=2$ and $b=3$, and one obtains (22) 
The second eigenvalue problem under consideration is a counterpart to Friedrichs' eigenvalue problem for pairs of conjugate harmonic functions. In dimension three, however, we introduce the real linear space $F$ consisting of all pairs $f=(p, q) \in$ $L^{2}(\Omega) \times L^{2}(\Omega)^{3}$ satisfying equations $(8),(9)$. Remember that those functions are harmonic in $\Omega$.

In the space $F$ we define a scalar product and norm by

$$
\left\langle f_{1}, f_{2}\right\rangle_{F}=\int_{\Omega}\left(p_{1} p_{2}+q_{1} \cdot q_{2}\right) d V \quad \text { and } \quad\|f\|_{F}^{2}=\int_{\Omega}\left(p^{2}+|q|^{2}\right) d V .
$$

Clearly, $\left(F,\langle\cdot,\rangle_{F},\|\cdot\|_{F}\right)$ is a Hilbert space. Indeed, any sequence $\left\{f_{k}\right\}$ satisfying $\| f_{j}-$ $f_{k} \|_{F} \rightarrow 0$ as $j, k \rightarrow \infty$ is convergent in $L^{2}(\Omega) \times L^{2}(\Omega)^{3}$. But as the functions $f_{k}=$ $\left(p_{k}, q_{k}\right)$ are solutions of equations (8), (9), the same holds for the limit function $f=$ $(p, q)$.

Besides of the quadratic functional $\|f\|_{F}^{2}$ we introduce also the quadratic functional

$$
Q_{F}(f)=\int_{\Omega}\left(p^{2}-|q|^{2}\right) d V
$$

and we ask for stationary values of the quotient $Q_{F}(f) /\|f\|_{F}^{2}$. Thus we are led to the variational eigenvalue problem of finding functions $f=(p, q) \in F \quad(f \neq 0)$ and real numbers $\mu$ such that

$$
\int_{\Omega}(p \tilde{p}-q \cdot \tilde{q}) d V=\mu \int_{\Omega}(p \tilde{p}+q \cdot \tilde{q}) d V \quad \text { for all }(\tilde{p}, \tilde{q}) \in F,
$$

where clearly $|\mu| \leq 1$. There are two eigenvalues which are independent of the shape of $\Omega$, namely $\mu=1$ and $\mu=-1$. The eigenspace to $\mu=1$ consists of all pairs $p=$ const, $q=0$, and the eigenspace to $\mu=-1$ of all pairs $p=0, q=\nabla h, h \in$ $H^{1}, \Delta h=0$. Any eigenfunction $f=(p, q)$ to an eigenvalue $\mu \neq \pm 1$ is orthogonal to the latter eigenspaces and satisfies therefore the conditions $p \neq 0$ and $q \neq 0$ together with $p \in L_{0}^{2}(\Omega)$ and $q \subset H_{0}(\operatorname{div} 0, \Omega)$.

If we restrict our attention to eigenvalues $\mu \neq \pm 1$, then we are led to the following

Problem II. Find funtions $f=(p, q) \in F \quad(f \neq 0)$ whith $p \in L_{0}^{2}(\Omega)$ and $q \in$ $H_{0}(\operatorname{div} 0, \Omega)$ such that

$$
\int_{\Omega} p \tilde{p} d V=\gamma \int_{\Omega} q \cdot \tilde{q} d V \quad \text { for all }(\tilde{p}, \tilde{q}) \in F,
$$

where $\gamma=\frac{1+\mu}{1-\mu}$ and $\gamma>0$.

Theorem 3. To any eigenvalue $\lambda$ of Problem $I$ other than $\lambda=1$ and $\lambda=\infty$ there is an eigenvalue $\gamma>0$ of Problem II such that

$$
\lambda=\gamma+1
$$

and vice versa. If $w$ is eigenfunction to $\lambda$, then the pair $p, q$ given by

$$
p=(\lambda-1) \operatorname{div} w \quad \text { and } \quad q=\operatorname{rot} w
$$


is solution of equation (24) to the eigenvalue $\gamma=\lambda-1$. Conversely, if the pair $p, q$ is solution of equation (24) to the eigenvalue $\gamma>0$, then there is an eigenfunction $w$ of (16) to the eigenvalue $\lambda=\gamma+1$ satisfying (25). The related eigenvalues $\gamma>0$ and $\lambda=\gamma+1$ are of the same multiplicity.

Proof. Let $w, \lambda$ denote a given eigensolution of Problem I where $\lambda \neq 1$ and $\lambda \neq \infty$ so that $w \in W$ by Proposition 5. On setting

$$
p=(\lambda-1) \operatorname{div} w \quad \text { and } \quad q=\operatorname{rot} w
$$

we obtain from (18) equations (8), (9), namely

$$
\int_{\Omega} q \cdot \operatorname{rot} \phi d V=\int_{\Omega} p \operatorname{div} \phi d V \quad \text { for all } \phi \in C_{0}^{\infty}(\Omega)^{3}
$$

and

$$
\int_{\Omega} q \cdot \nabla \Phi d V=0 \quad \text { for all } \Phi \in C_{0}^{\infty}(\Omega)
$$

Thus the pair $(p, q)$ belongs to the space $F$. Note that $p \in \operatorname{div} W \subset L_{0}^{2}(\Omega)$ and $q \in$ rot $W \subset H_{0}$ (div $\left.0, \Omega\right)$ by Proposition 2. Since, however, $C_{0}^{\infty}(\Omega)^{3}$ is dense in $H_{0}^{1}(\Omega)^{3}$, equation (8) is satisfied for $\phi=w$, too. Thus we obtain for any pair $(\tilde{p}, \tilde{q}) \in F$ the relation

$$
\int_{\Omega} \tilde{p} \operatorname{div} w d V=\int_{\Omega} \tilde{q} \cdot \operatorname{rot} w d V
$$

and in view of (26)

$$
\int_{\Omega} p \tilde{p} d V=(\lambda-1) \int_{\Omega} q \cdot \tilde{q} d V \quad \text { for all }(\tilde{p}, \tilde{q}) \in F .
$$

Hence the pair $(p, q)$ defined by $(26)$ is eigensolution of Problem II to the eigenvalue $\gamma=\lambda-1>0$.

Conversely, let $(p, q) \in F$ be eigensolution of Problem II to the eigenvalue $\gamma>0$. By Proposition 4 there holds $p \in \operatorname{div} W$ and $q \in \operatorname{rot} W$. In particular, there exists a uniquely defined function $w \in W$ so that $q=\operatorname{rot} w$. On inserting the latter function into equation (24) we obtain from (27) the equation

$$
\int_{\Omega}(p-\gamma \operatorname{div} w) \tilde{p} d V=0 \quad \text { for all } \tilde{p} \in \operatorname{div} W,
$$

whence $p=\gamma \operatorname{div} w$. On inserting the two functions $p=\gamma \operatorname{div} w$ and $q=\operatorname{rot} w$ into equation (8), we find that $w$ is solution of (18) to the eigenvalue $\lambda=\gamma+1$

An immediate consequence of Mikhlin's results quoted in Theorem 2 is a compactness result already stated in [10]. In our notation it may be formulated as follows:

If restricted to the subspace $W \in H_{0}^{1}(\Omega)^{3}$, the quadratic functional

$$
Q_{w}(w)=r(w)-d(w)
$$


is compact with respect to $d(w)$. This means, given in $W$ a sequence $\left\{w^{(k)}\right\}$ bounded in the sense $d\left(w^{(k)}\right) \leq M$ and converging weakly to zero, then

$$
Q_{W}\left(w^{(k)}\right) \rightarrow 0 \quad \text { as } k \rightarrow \infty .
$$

This is easily seen as follows. As in the proof of Theorem 3 we introduce in $W$ a complete orthogonal system $\left\{w_{n}\right\}$ of eigenfunctions normalized by $d\left(w_{n}\right)=1$ and numbered such that $\left|\lambda_{n}-2\right| \downarrow$. Any of the functions $w^{(k)}$ has therefore a representation

$$
w^{(k)}=\sum_{n} c_{n}^{(k)} w_{n} \quad \text { with } \quad c_{n}^{(k)}=d\left(w^{(k)}, w_{n}\right)
$$

where, due to the weak convergence to zero, $c_{n}^{(k)} \rightarrow 0$ as $k \rightarrow \infty$. Thus one obtains

$$
r\left(w^{(k)}\right)-d\left(w^{(k)}\right)=\sum_{n}\left(\lambda_{n}-2\right)\left(c_{n}^{(k)}\right)^{2} \quad \text { and } \quad d\left(w^{(k)}\right)=\sum_{n}\left(c_{n}^{(k)}\right)^{2} \leq M
$$

where $\left(\lambda_{n}-2\right) \rightarrow 0$ as $n \rightarrow \infty$. Compactness follows now by a well known argument. Given an integer $N \geq 1$, one obtains the estimate

$$
\left|r\left(w^{(k)}\right)-d\left(w^{(k)}\right)\right| \leq \sum_{n \leq N}\left|\lambda_{n}-2\right|\left(c^{(k)}\right)^{2}+\left|\lambda_{N+1}-2\right| M .
$$

In view of $c_{n}^{(k)} \rightarrow 0$ as $k \rightarrow \infty$, the finite sum at the right-hand side tends to zero as $k \rightarrow \infty$. The term $\left|\lambda_{N+1}-2\right| M$, however, is as small as we want since $\left|\lambda_{N+1}-2\right| \rightarrow 0$ as $N \rightarrow \infty$.

Remark. Since $d(\cdot)^{1 / 2}$ and $r(\cdot)^{1 / 2}$ are equivalent norms in $W$, the quadratic form $Q_{W}(\cdot)$ is compact with respect to $r(\cdot)$, too.

Due to Theorem 3 there is a completeness and compactness result for Problem II as well. The counterpart to the subspace $W$ is now the subspace $G \subset F$ defined by

$$
G=\left\{(p, q) \in F \mid p \in L_{0}^{2}(\Omega) \text { and } q \in H_{0}(\operatorname{div} 0, \Omega)\right\}
$$

Theorem 4. In the subspace $G$ of the Hilbert space $\left(F,(\cdot, \cdot\rangle_{F},\|\cdot\|_{F}\right)$ there exists a complete orthogonal system $\left\{f_{n}\right\}$ of eigenfunctions $f_{n}=\left(p_{n}, q_{n}\right)$ to eigenvalues $\gamma_{n}>0$. If restricted to the subspace $G$, the quadratic form

$$
Q_{F}(f)=\int_{\Omega}\left(p^{2}-|q|^{2}\right) d V
$$

is compact with respect to each of the two forms $\|p\|^{2}=\int_{\Omega} p^{2} d V$ and $\|q\|^{2}=\int_{\Omega} \cdot|q|^{2} d V$.

Proof. Let $f=(p, q) \in G$ be given. Due to Proposition 2 there exist functions $w \in W$ and $\tilde{w} \in W$ such that $p=\operatorname{div} w$ and $q=\operatorname{rot} \tilde{w}$. Introducing representations in terms of eigenfunctions $w_{n}$ as in the proof of Theorem 3, namely

$$
w=\sum_{n} c_{n} w_{n} \quad \text { and } \quad \tilde{w}=\sum_{n} \tilde{c}_{n} w_{n}
$$


where $d\left(w_{n}\right)=1, c_{n}=d\left(w, w_{n}\right)$, and $\tilde{c}_{n}=d\left(\tilde{w}, w_{n}\right)$, we obtain

$$
p=\sum_{n} c_{n} \operatorname{div} w_{n} \quad \text { and } \quad q=\sum_{n} \tilde{c}_{n} \operatorname{rot} w_{n}
$$

Since, however, $p$ and $q$ satisfy equation (8) for any $\phi \in H_{0}^{1}(\Omega)$, we find in particular the relations

$$
c_{n}=\int_{\Omega} p \operatorname{div} w_{n} d V=\int_{\Omega} q \operatorname{rot} w_{n} d V=\tilde{c}_{n} \int_{\Omega}\left|\operatorname{rot} w_{n}\right|^{2} d V=\left(\lambda_{n}-1\right) \tilde{c}_{n} .
$$

At the other hand, the pairs $p_{n}, q_{n}$ defined by $p_{n}=\left(\lambda_{n}-1\right) \operatorname{div} w_{n}$ and $q_{n}=\operatorname{rot} w_{n}$ are eigenfunctions of Problem II to the eigenvalues $\gamma_{n}=\lambda_{n}-1$. Hence we obtain

$$
p=\sum_{n} \tilde{c}_{n} p_{n}, \quad q=\sum_{n} \tilde{c}_{n} q_{n}, \quad \text { or } \quad f=\sum_{n} \tilde{c}_{n} f_{n} .
$$

That is, any $f \in G$ has a representation in terms of the orthogonal eigenfunctions $f_{n}=\left(p_{n}, q_{n}\right)$ to eigenvalues $\gamma_{n}>0$. On inserting the series into the functional $Q_{F}(\cdot)$ we find

$$
Q_{F}(f)=\sum_{n}\left(1-\gamma_{n}^{-1}\right) c_{n}^{2} \quad \text { and } \quad\|p\|^{2}=\sum_{n} c_{n}^{2}
$$

Since, however, $\gamma_{n} \rightarrow 1$ and $\left(1-\gamma_{n}^{-1}\right) \rightarrow 0$ as $n \rightarrow \infty$, the quadratic form $Q_{F}(f)$ is indeed compact with respect to the quadratic form $\|p\|^{2}$. Due to Theorem, $I$, however, the form $\|p\|^{2}$ may be replaced by the form $\|q\|^{2}$

\section{An inequality related with the Stokes equations}

Given a bounded domain $\Omega$, the first boundary value problem for the Stokes equations reads

$$
\left.\begin{array}{rl}
-\Delta u+\nabla p=f & \\
\operatorname{div} u=0 & \text { in } \Omega \\
u=0 & \text { on } \partial \Omega
\end{array}\right\}
$$

where $p$ may be normalized by $\int_{\Omega} p d V=0$.

When dealing with numerical algorithms for solving the Stokes problem in weak (variational) form, the following inequality between two norms of the pressure function $p \in L_{0}^{2}(\Omega)$ plays an important role:

$$
\|p\|^{2} \leq C^{*} \sup _{\phi \in H_{0}^{1}(\Omega)^{3}} \frac{(\operatorname{div} \phi, p)^{2}}{D(\phi)} .
$$

Here $(\cdot, \cdot)$ and $\|\cdot\|$ denote the usual scalar product and norm in $L^{2}(\Omega)$.

There is a simple relation between the optimal constant $C^{*}$ in (29) and the optimal constant $C$ introduced in (6), namely

$$
C^{*}=C \text {. }
$$


This result is already stated in the lecture note [12]. The constant $C^{*}$ controls in particular the convergence speed of certain algorithms, so that it is of interest to have informations on it. Therefore we will add here a proof of $(30)$.

(i) $C^{*} \leq C$ : Let $p \in L_{0}^{2}(\Omega)$ be given. Due to Proposition 1 and decomposition (5) there exists $u \in H_{0}^{1}(\Omega)^{3}, u \in \operatorname{Ker} \operatorname{div}^{\perp}$ such that $p=\operatorname{div} u$. From inequality (6) we infer

$$
\frac{(\operatorname{div} u, p)^{2}}{D(u)}=\frac{\|\operatorname{div} u\|^{2}}{D(u)}\|p\|^{2} \geq \frac{1}{C}\|p\|^{2}
$$

whence

$$
\|p\|^{2} \leq C \sup _{\phi \in H_{0}^{2}(\Omega)^{3}} \frac{(\operatorname{div} \phi, p)^{2}}{D(\phi)} \quad \text { for all } p \in L_{0}^{2}(\Omega),
$$

that is $C^{*} \leq C$.

(ii) $C=C^{*}$ : In order to exclude the strict inequality $C^{*}<C$, it is sufficient to consider functions $p$ defined by $p=\operatorname{div} u_{k}$, where $u_{k} \in W$ is eigenfunction to the eigenvalue $\lambda_{k}$,

$$
D\left(u_{k}, \phi\right)=\lambda_{k} d\left(u_{k}, \phi\right) \quad \text { for all } \phi \in H_{0}^{1}(\Omega)^{3} .
$$

Let $u_{k}$ be normalized by $d\left(u_{k}, u_{k}\right)=1$. For those functions $p$ it is possible to evaluate the supremum in (29) explicitly. Indeed, any given $\phi \in H_{0}^{1}(\Omega)^{3}, \phi \neq 0$, has a representation $\phi=\alpha u_{k}+\phi^{\perp}$ where the two components are, by construction, $D$-orthogonal and then due to (31) $d$-orthogonal, too. Thus we obtain

$$
(\operatorname{div} \phi, p)^{2}=\left(\alpha \operatorname{div} u_{k}, p\right)^{2}=\alpha^{2} \quad \text { and } \quad D(\phi) \geq D\left(\alpha u_{k}\right)=\alpha^{2} \lambda_{k} .
$$

The supremum in (29) is attained if and only if $\phi=\alpha \operatorname{div} u_{k} \quad(\alpha \neq 0)$ so that

$$
1 \leq C^{*} \sup _{\phi \in H_{0}^{1}(\Omega)^{3}} \frac{(\operatorname{div} \phi, p)^{2}}{D(\phi)\|p\|^{2}}=\frac{C^{*}}{\lambda_{k}} \quad \text { or } \quad \lambda_{k} \leq C^{*} .
$$

But due to Theorem 3, the constant $C$ is the lowest upper bound to the eigenvalues $\lambda_{k}$ belonging to eigenfunctions $u_{k} \in W$, whence $C \leq C^{*}$. This completes the proof

Let us also notice that inequality (29) has a counterpart where div is replaced by rot and $p$ by $q$, and where $(\cdot, \cdot)$ and $\|\cdot\|$ denote now the inner product and norm in $L^{2}(\Omega)^{3}$. It reads as follows:

There is a constant $\tilde{C}^{*}$ depending only on $\Omega$ such that for any $q \in H_{0}(\operatorname{div} 0, \Omega)$

$$
\|q\|^{2} \leq \tilde{C}^{*} \sup _{\phi \in H_{0}^{1}(\Omega)^{3}} \frac{(\operatorname{rot} \phi, q)^{2}}{D(\phi)} .
$$

The optimal constant in (32) is given by

$$
\tilde{C}^{*}=\tilde{C} \text {. }
$$

The proof of (33) parallels the proof of (30). For given $q$ there exists $u \perp$ Ker rot such that $q=\operatorname{rot} u$, which yields $\tilde{C}^{*} \leq \tilde{C}$. The eigensolution $u_{k}, \lambda_{k}$ has to be replaced by the eigensolution $u_{k}, \rho_{k}$ of

$$
D\left(u_{k}, \phi\right)=\rho_{k} r\left(u_{k}, \phi\right) \quad \text { for all } \phi \in H_{0}^{1}(\Omega)^{3},
$$

where $\rho_{k}=1+\left(\lambda_{k}-1\right)^{-1}$. It follows $\rho_{k} \leq \tilde{C}^{*}$ and hence $\tilde{C} \leq \tilde{C}^{*}$ as well. 
Acknowledgements. It is a pleasure to acknowledge various stimulating discussions with M. Dobrowolski and C. G. Simader on the material presented above.

\section{References}

[1] Babuška,I. und A. K. Aziz: Survey lectures on the mathematical fundation of the finite element method. In: The Mathematical Foundation of the Finite Element Method with Applications to Partial Differential Equations (ed.: A. K. Aziz). New York: Acad. Press 1972, Chapter 5, pp. $111-184$.

[2] Cosserat, E. and F. Cosserat: Sur les équations de la théorie de l'élasticité. C.R. Acad. Sci. Paris 126 (1898), 1089 - 1091.

[3] Dautray, R. and J.-L. Lions: Mathematical Analysis and Numerical Methods for Science and Technology. Berlin: Springer-Verlag 1990.

[4] Friedrichs, K. O.: On certain inequalities and characteristic value problems for analytic functions of two variables. Trans. Amer. Math. Soc. 41 (1937), $321-364$.

[5] Girault, V. and P.-A. Raviart: Finite Element Methods for Navier-Stokes Equations. Berlin: Springer-Verlag 1986.

[6] Horgan, C. O. and L. E. Payne: On inequalities of Korn, Friedrichs and Babuška-Aziz. Arch. Rat. Mech. Anal. 82 (1983), 165 - 179.

[7] Horgan, C. O.: Korn's inequalities and their applications in continuum mechanics. SIAM Review 37 (1995), 491 - 511.

[8] Horgan, C. O.: Inequalities of Korn and Friedrichs in elasticity and potential theory. J. Appl. Math. Phys. (ZAMP) 26 (1975), $155-164$.

[9] Ladyzhenskaya, O. A. and V. A. Solonnikov: Some problems of vector analysis and generalized formulations of boundary-value problems for the Navier-Stokes equations. Soviet Math. 10 (1978), 257 - 286.

[10] Mikhlin, S. G.: The spectrum of a family of operators in the theory of elasticity. Russ. Math. Surveys 28 (1973)3, $43-82$.

(11] Velte, W.: On inequalities of Friedrichs and Babuška-Aziz. Meccanica 31 (1996), 589 596.

[12] Velte, W.: On optimal constants in some inequalities. Lect. Notes Math. 1431 (1990), $158-168$. 